\title{
Chatter Prediction for Variable Pitch and Variable Helix Milling
}

\author{
Yong Wang, ${ }^{1,2}$ Taiyong Wang, ${ }^{1}$ Zhiqiang Yu, ${ }^{1}$ Yue Zhang, ${ }^{1}$ Yulong Wang, ${ }^{1}$ and Hengli Liu ${ }^{1,2}$ \\ ${ }^{1}$ Key Laboratory of Mechanism Theory and Equipment Design of Ministry of Education, Tianjin University, Tianjin 300072, China \\ ${ }^{2}$ School of Mechanical Engineering, Tianjin University of Commerce, Tianjin 300134, China \\ Correspondence should be addressed to Yong Wang; wylxl2001@163.com
}

Received 6 January 2015; Revised 4 April 2015; Accepted 6 April 2015

Academic Editor: Tony Murmu

Copyright (C) 2015 Yong Wang et al. This is an open access article distributed under the Creative Commons Attribution License, which permits unrestricted use, distribution, and reproduction in any medium, provided the original work is properly cited.

\begin{abstract}
Regenerative chatter is a self-excited vibration that can occur during milling, which shortens the lifetime of the tool and results in unacceptable surface quality. In this paper, an improved semidiscretization method for modeling and simulation with variable pitch and variable helix milling is proposed. Because the delay between each flute varies along the axial depth of the tool in milling, the cutting tool is discrete into some axial layers to simplify calculation. A comparison of the predicted and observed performance of variable pitch and variable helix against uniform pitch and uniform helix milling is presented. It is shown that variable pitch and variable helix milling can obtain larger stable cutting area than uniform pitch and uniform helix milling. Thus, it is concluded that variable pitch and variable helix milling are an effective way for suppressing chatter.
\end{abstract}

\section{Introduction}

In the present day, an increasing demand for high-precision products is posed in manufacturing industry. However, the main obstacles in achieving high productivity derive from periodical excitation vibrations which resulted from the dynamic cutting forces between the cutter and the workpiece in milling. This vibration is the so-called self-excited chatter. It is well known that chatter affects adversely both surface finish and dimensional accuracy of the workpiece. Furthermore, it increases tool wear and may cause tool fracture and damage to the machine tool itself. Thus, how to use effective means to avoid these harmful phenomena has become critical. In the long-term studies, various approaches have been developed in this field, which include numerical, analytical, and experimental ones. These results have become the foundation of theoretical study.

To predict the stability boundaries related to chatter, an accurate dynamic model for the milling process is required. All kinds of models have been suggested since the late 1950 s. When dealing with related problems, the stability lobe diagram (SLD) based on regenerative chatter theory is widely accepted to predict and control chatter. In a SLD, the chatter stability boundary between a stable cut (i.e., without chatter) and an unstable cut (i.e., with chatter) can be visualized in terms of spindle speed and depth of cut. Altintaş [1,2], as an expert in machine dynamics, presented an analytical method of predicting stability limits in milling. This method is based on the formulation of dynamic milling with regeneration in the interaction with the machine tool structure to obtain stability lobes in the frequency domain. Although the zeroorder solution can receive reasonably accurate predictions mostly, it may fail to capture added stability lobes in special conditions, that is, highly intermittent machining or at low radial immersion milling. Hence, Merdol and Altintas [3] proposed multifrequency solution by considering a number of harmonics of directional coefficients to solve this issue. In addition, the time-domain simulation is considered to be a good choice for accurate stability predictions in milling. The semidiscretization method (SDM) is firstly applied to milling by Insperger and Stepan [4-6]; SDM can correctly solve the problem of milling stability under different conditions and be easily implemented on the computer, so it is widely employed. Bayly et al. [7] implemented time finite element analysis (TFEA) to obtain stability map of intermittent machining. This method was used to form an approximate solution at each time increment in the cut and checks each cutting condition by time finite analysis and uses simulations to determine whether it is stable or not. Ding et al. [8] presented a full-discretization method based on the direct integration 
scheme for prediction of milling stability. It shows that the computational times can be reduced by about $60-75 \%$ in comparison with SDM without loss of any numerical precision. Furthermore, the D-partition method [9], the Lambert $W$ function based method [10], and the Chebyshev collocation method [11] are also effective numerical methods for predicting the stability of milling.

It is noteworthy that the influence of helix angle is not considered in the above works, which will lead to some important dynamic properties being ignored. Zatarain et al. [12] pointed out that helix angle of the mill can have an important role in instability due to repetitive impact driven chatter (flip). Through the development of multifrequency solution, they thought that the flip lobes became closed curves which are separated by horizontal lines where the depth of cut equals a multiple of the helix pitch. A similar phenomenon was confirmed by using SDM in Insperger's literature [13]. Altintas [2] proposed that the lower and upper limits of integration for every helical flute can be divided into five different cases when the cutting force model for helix milling is established. Based on this idea, Patel et al. [14] considered the influence of the tool helix angle to obtain an analytical force model with three piecewise continuous regions of cutting and provided conclusive evidence that isolated islands of chatter vibration can exist in milling processes. Investigations showed that these islands were induced by the tool helix angle and led to separate regions of period-doubling and quasiperiod behavior. We know that these results are established on the basis of the cutters with uniform helix angle and uniform pitch. In milling process, chatter can also be improved by changing the pitch and/or helix angle of the cutters. For variable pitch cutters, the theory of zero-order solution was modified to cover this case by Altintaş et al. [15]. An analytical model was presented by Budak $[16,17]$ for variable pitch cutters to determine the optimal pitch angles. A novel mathematical approach, the cluster treatment of characteristic root (CTCR), was proposed by Olgac and Sipahi [18], which enables the optimal design of variable pitch cutters. For variable helix cutters, a new analytical model was presented by Turner et al. [19], to inquire into the kinematics of milling. They showed the results acceptable when the axial engagement of the tool was low so that the variable pitch approximation remained valid. Yusoff and Sims [20] combined SDM with differential evolution to optimize variable helix end milling tools for minimising chatter. The analysis predicted total mitigation of chatter using the optimised variable helix milling tool at a low radial immersion.

A review of the literature shows that few works focused on the combined variable helix and variable pitch cutters. One exception is the works by Sims et al. [21]. For variable helix tools the delay between each flute varies along the axial depth of the tool; they deemed that this can be tackled by discretising the tool into a number of axial layers and discretising in the time domain so that $N$ time steps occur in one tool revolution. This paper will take the above idea to simplify calculation. Based on the results of the former research studies $[5,8,21]$, we proposed an improved SDM to analyze the stability of variable pitch and variable helix

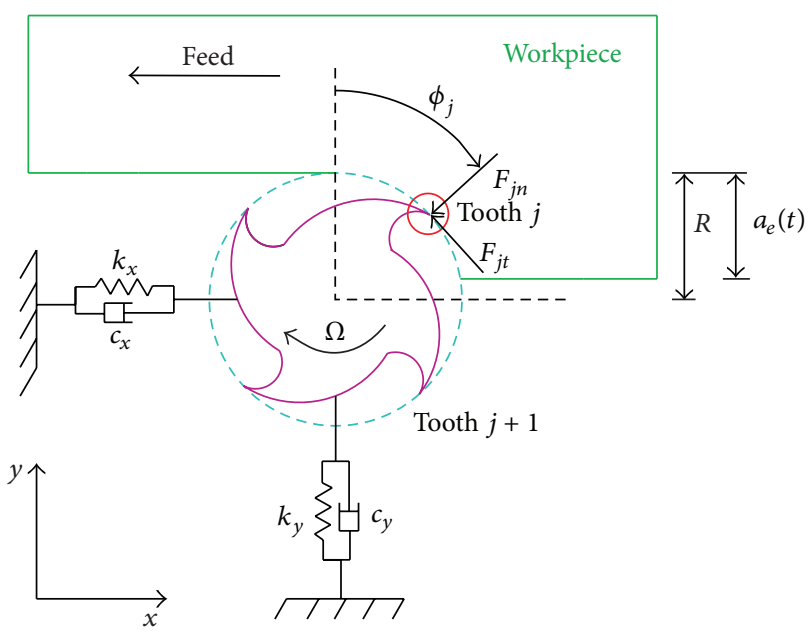

FIGURE 1: Schematic mechanical model of the milling process.

milling. This paper is organized as follows. In Section 2, the mathematical model is introduced. In Section 3, the numerical algorithms are described in detail. In Section 4, 2-DOF down-milling model is provided to illustrate the improved method. In Section 5, conclusions with a brief discussion are presented.

\section{The Dynamic Milling Model}

A schematic diagram of the two-degree-of-freedom system is shown in Figure 1. The mathematical representation of this system is given by

$$
\begin{aligned}
& m_{x} \ddot{x}+c_{x} \dot{x}+k_{x} x=F_{x}(t), \\
& m_{y} \ddot{y}+c_{y} \dot{y}+k_{y} y=F_{y}(t),
\end{aligned}
$$

where $m_{q}, c_{q}$, and $k_{q}(q=x, y)$ are the mass, damping, and stiffness parameters, respectively. Due to the helix of the tool (the difference between uniform and variable helix and pitch tools as illustrated in Figures 2 and 3), the cutting force in $x$ and $y$ directions can be written as

$$
\begin{aligned}
& F_{x}=\sum_{j=1}^{N} \sum_{l=1}^{L} f_{x, l, j}(t), \\
& F_{y}=\sum_{j=1}^{N} \sum_{l=1}^{L} f_{y, l, j}(t),
\end{aligned}
$$

where $N$ is the number of teeth and $L$ is the number of axial layers. The $x$ and $y$ components of the cutting force for the tooth $j$ on the layer $l$ at time $t$ are

$$
\begin{aligned}
f_{x, l, j}(t)= & -f_{t, l, j}(t) \cos \left(\phi_{l, j}(t)\right) \\
& -f_{n, l, j}(t) \sin \left(\phi_{l, j}(t)\right), \\
f_{y, l, j}(t)= & f_{t, l, j}(t) \sin \left(\phi_{l, j}(t)\right)-f_{n, l, j}(t) \cos \left(\phi_{l, j}(t)\right) .
\end{aligned}
$$




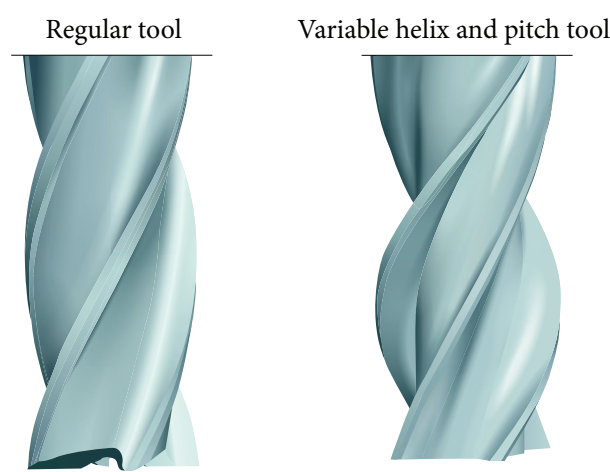

Figure 2: 3D virtual models.

The angular position of the $j$ th tooth of the $l$ th axial layer is defined by

$$
\phi_{l, j}(t)=\frac{2 \pi \Omega}{60} t+p_{j}-z \frac{2 \tan \beta_{j}(l-1)}{d_{c} L},
$$

where $\Omega(\mathrm{rpm})$ is the spindle speed, $p_{j}$ is the pitch between the $j$ th tooth and the $(j+1)$ th tooth, $\beta_{j}$ is the helix angle of the $j$ th tooth, $d_{c}$ is the diameter of the tool, and $z$ is the axial depth of cut. The tangential and the normal forces for the tooth $j$ on the layer $l$ can be expressed by

$$
\begin{aligned}
& f_{t, l, j}(t)=g\left(\phi_{l, j}(t)\right) K_{t} h_{l, j}(t) \Delta z, \\
& f_{n, l, j}(t)=g\left(\phi_{l, j}(t)\right) K_{n} h_{l, j}(t) \Delta z,
\end{aligned}
$$

where $\Delta z=z / L$ and $K_{t}$ and $K_{n}$ are tangential and normal cutting coefficients, respectively. $g\left(\phi_{l, j}(t)\right)$ is the window function; it equals one if the tooth $j$ at layer $l$ is in the cut or equals zero when out of cut:

$$
g\left(\phi_{l, j}(t)\right)= \begin{cases}1 & \text { if } \phi_{s t}<\phi_{l, j}(t)<\phi_{e x} \\ 0 & \text { otherwise. }\end{cases}
$$

Here, $\phi_{s t}$ and $\phi_{e x}$, respectively, define the angles at which the teeth enter and leave the workpiece. The dynamic chip thickness caused by vibration is

$$
\begin{aligned}
h_{l, j}(t)= & \left(x(t)-x\left(t-\tau_{l, j}\right)\right) \sin \left(\phi_{l, j}(t)\right) \\
& +\left(y(t)-y\left(t-\tau_{l, j}\right)\right) \cos \left(\phi_{l, j}(t)\right),
\end{aligned}
$$

where $\tau_{l, j}$, the time delay, means that the current tooth at the present time $t$ is to remove the surface waves which was generated by the previous tooth at time $t-\tau_{l, j}$ and time delay $\tau_{l, j}$ can be described by

$$
\tau_{l, j}=T \frac{\Delta \phi_{l, j}}{2 \pi},
$$

where $T=60 / \Omega$ is time period; the pitch between one tooth and the previous one is given by

$$
\Delta \phi_{l, j}= \begin{cases}\phi_{l, j+1}(t)-\phi_{l, j}(t) & j<N, \\ \phi_{l, 1}(t)-\phi_{l, j}(t)+2 \pi & j=N .\end{cases}
$$

According to the above analysis, the governing equation of milling process can be obtained. In the following, we will propose the improved SDM to deal with variable pitch and variable helix milling issue.

\section{Numerical Algorithms}

Let $\mathbf{q}(t)=(x(t), y(t))^{T}, \mathbf{p}(t)=\mathbf{M} \dot{\mathbf{q}}+\mathbf{C q} / 2$, and $\mathbf{y}(t)=$ $(\mathbf{q}(t), \mathbf{p}(t))^{T}$. Through algebraic transformations, (1) can be represented by

$$
\begin{aligned}
\dot{\mathbf{y}}(t)= & \mathbf{A}_{0} \mathbf{y}(t) \\
& +\sum_{j=1}^{N} \sum_{l=1}^{L}\left[\mathbf{A}_{l, j}(t) \mathbf{y}(t)-\mathbf{B}_{l, j}(t) \mathbf{y}\left(t-\tau_{l, j}\right)\right],
\end{aligned}
$$

with

$$
\begin{aligned}
\mathbf{A}_{0}= & \left(\begin{array}{cc}
\frac{-\mathbf{M}^{-1} \mathbf{C}}{2} & \mathbf{M}^{-1} \\
\frac{\mathbf{C} \mathbf{M}^{-1} C}{4}-\mathbf{K} & \frac{-\mathbf{C M}^{-1}}{2}
\end{array}\right), \\
\mathbf{A}_{l, j}(t) & =\mathbf{B}_{l, j}(t)=\left(\begin{array}{cccc}
0 & 0 & 0 & 0 \\
0 & 0 & 0 & 0 \\
h_{x x}(t) & h_{x y}(t) & 0 & 0 \\
h_{y x}(t) & h_{y y}(t) & 0 & 0
\end{array}\right),
\end{aligned}
$$

where

$$
\begin{aligned}
& h_{x x}(t)=-g\left(\phi_{l, j}(t)\right) \\
& \cdot {\left[K_{t} \cos \left(\phi_{l, j}(t)\right)+K_{n} \sin \left(\phi_{l, j}(t)\right)\right] } \\
& \cdot \sin \left(\phi_{l, j}(t)\right) \Delta z, \\
& h_{x y}(t)=-g\left(\phi_{l, j}(t)\right) \\
& \cdot\left[K_{t} \cos \left(\phi_{l, j}(t)\right)+K_{n} \sin \left(\phi_{l, j}(t)\right)\right] \\
& \cdot \cos \left(\phi_{l, j}(t)\right) \Delta z, \\
& h_{y x}(t)=-g\left(\phi_{l, j}(t)\right) \\
& \cdot\left[-K_{t} \sin \left(\phi_{l, j}(t)\right)+K_{n} \cos \left(\phi_{l, j}(t)\right)\right] \\
& \cdot \sin \left(\phi_{l, j}(t)\right) \Delta z, \\
& h_{y y}(t)=-g\left(\phi_{l, j}(t)\right) \\
& \cdot\left[-K_{t} \sin \left(\phi_{l, j}(t)\right)+K_{n} \cos \left(\phi_{l, j}(t)\right)\right] \\
& \cdot \cos \left(\phi_{l, j}(t)\right) \Delta z .
\end{aligned}
$$

The first step of semidiscretization is the construction of the time interval division of $\left[t_{i}, t_{i+1}\right]$ of length $\Delta t, i=0,1, \ldots$, so that $T=k \Delta t$, where $k$ is an integer that can be considered as an approximation parameter regarding the time period. 


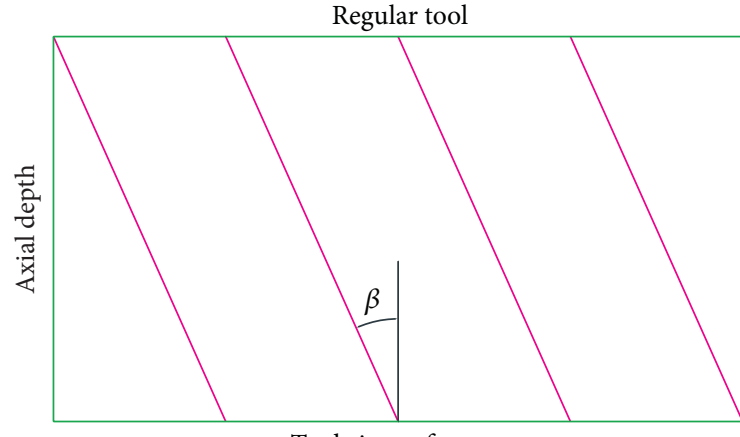

Tool circumference

(a)

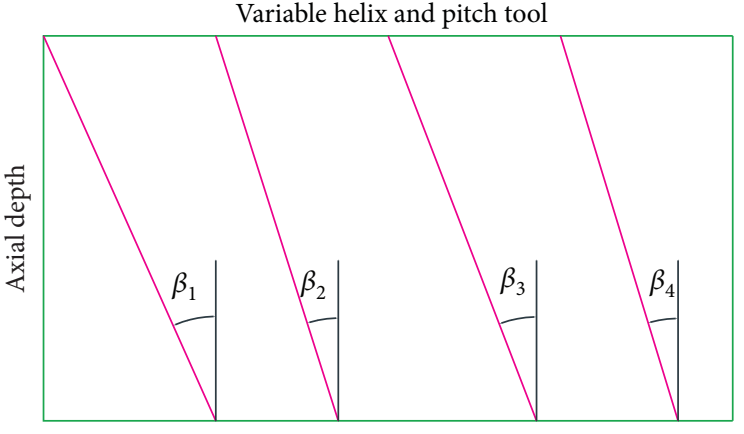

Tool circumference

(b)

FIgURE 3: The expanded views of the blade edge.

The integers $m_{l, j}$ related to the delay $\tau_{l, j}$ can be approximately obtained by

$$
m_{l, j}=\operatorname{int}\left(\frac{\tau_{l, j}+\Delta t / 2}{\Delta t}\right),
$$

where $\operatorname{int}(*)$ is the function that rounds positive numbers towards zero (e.g., $\operatorname{int}(3.17)=3$ ). Since integer $m_{l, j}$ might be different for different semidiscretization steps, we introduce the maximum value of $m_{l, j}$ as $M=\max _{l, j=1,2, \ldots}\left\{m_{l, j}\right\}$.

Then, solving (10) as an ordinary differential equation over the discretization period $\left[t_{i}, t_{i+1}\right]$ with initial condition $\mathbf{y}\left(t_{i}\right)=\mathbf{y}_{i}$, we can obtain

$$
\begin{aligned}
& \mathbf{y}(t)=e^{\mathbf{A}_{0}\left(t-t_{i}\right)} \mathbf{y}_{i} \\
& \quad+\int_{t_{i}}^{t}\left\{e^{\mathbf{A}_{0}(t-\xi)} \sum_{j=1}^{N} \sum_{l=1}^{L}\left[\mathbf{A}_{l, j}(\xi) \mathbf{y}(\xi)-\mathbf{B}_{l, j}(\xi) \mathbf{y}\left(\xi-\tau_{l, j}\right)\right]\right\} \mathrm{d} \xi .
\end{aligned}
$$

Equation (14) can be equivalently expressed as

$$
\begin{aligned}
& \mathbf{y}\left(t_{i+1}\right)=e^{\mathbf{A}_{0} \Delta t} \mathbf{y}_{i} \\
& \quad+\int_{t_{i}}^{t_{i+1}}\left\{e^{\mathbf{A}_{0}\left(t_{i+1}-\xi\right)} \sum_{j=1}^{N} \sum_{l=1}^{L}\left[\mathbf{A}_{l, j}(\xi) \mathbf{y}(\xi)-\mathbf{B}_{l, j}(\xi) \mathbf{y}\left(\xi-\tau_{l, j}\right)\right]\right\} \mathrm{d} \xi,
\end{aligned}
$$

where $t_{i}=i \Delta t$ (with $i \in Z$ ) and

$$
\begin{gathered}
\mathbf{A}_{l, j}(t)=\mathbf{A}_{i}^{\langle l, j\rangle}+\frac{\mathbf{A}_{i+1}^{\langle l, j\rangle}-\mathbf{A}_{i}^{\langle l, j\rangle}}{\Delta t}\left(t-t_{i}\right), \\
\mathbf{B}_{l, j}(t)=\mathbf{B}_{i}^{\langle l, j\rangle}+\frac{\mathbf{B}_{i+1}^{\langle l, j\rangle}-\mathbf{B}_{i}^{\langle l, j\rangle}}{\Delta t}\left(t-t_{i}\right), \\
\mathbf{y}(t)=\mathbf{y}_{i}+\frac{\mathbf{y}_{i+1}-\mathbf{y}_{i}}{\Delta t}\left(t-t_{i}\right), \\
\mathbf{y}\left(t-\tau_{l, j}\right)=\mathbf{y}_{i-m_{l, j}}+\frac{\mathbf{y}_{i+1-m_{l, j}}-\mathbf{y}_{i-m_{l, j}}}{\Delta t}\left(t-t_{i}\right),
\end{gathered}
$$

Substituting (16) into (15) leads to

$$
\begin{aligned}
\mathbf{y}_{i+1}= & \left(\boldsymbol{\Phi}_{0}+\sum_{j=1}^{N} \sum_{l=1}^{L} \mathbf{F}_{i}^{\langle l, j\rangle}\right) \mathbf{y}_{i}+\sum_{j=1}^{N} \sum_{l=1}^{L} \mathbf{P}_{i}^{\langle l, j\rangle} \mathbf{y}_{i+1} \\
& -\sum_{j=1}^{N} \sum_{l=1}^{L} \mathbf{F}_{i}^{\langle l, j\rangle} \mathbf{y}_{i-m_{l, j}}-\sum_{j=1}^{N} \sum_{l=1}^{L} \mathbf{P}_{i}^{\langle l, j\rangle} \mathbf{y}_{i+1-m_{l, j}},
\end{aligned}
$$

where

$$
\begin{aligned}
\mathbf{F}_{i}^{\langle l, j\rangle}= & \left(\boldsymbol{\Phi}_{1}-\frac{2}{\Delta t} \boldsymbol{\Phi}_{2}+\frac{1}{\Delta t^{2}} \boldsymbol{\Phi}_{3}\right) \mathbf{A}_{i}^{\langle l, j\rangle} \\
& +\left(\frac{1}{\Delta t} \boldsymbol{\Phi}_{2}-\frac{1}{\Delta t^{2}} \boldsymbol{\Phi}_{3}\right) \mathbf{A}_{i+1}^{\langle l, j\rangle}, \\
\mathbf{P}_{i}^{\langle l, j\rangle}= & \left(\frac{1}{\Delta t} \boldsymbol{\Phi}_{2}-\frac{1}{\Delta t^{2}} \boldsymbol{\Phi}_{3}\right) \mathbf{A}_{i}^{\langle l, j\rangle}+\left(\frac{1}{\Delta t^{2}} \boldsymbol{\Phi}_{3}\right) \mathbf{A}_{i+1}^{\langle l, j\rangle} .
\end{aligned}
$$

Clearly, $\Phi_{0}, \Phi_{1}, \Phi_{2}$, and $\Phi_{3}$ can be expressed as follows:

$$
\begin{aligned}
& \boldsymbol{\Phi}_{0}=e^{\mathbf{A}_{0} \Delta t}, \\
& \boldsymbol{\Phi}_{1}=\int_{0}^{\Delta t} e^{\mathbf{A}_{0}(\Delta t-s)} \mathrm{d} s=\mathbf{A}_{0}^{-1}\left(\boldsymbol{\Phi}_{0}-\mathbf{I}\right), \\
& \boldsymbol{\Phi}_{2}=\int_{0}^{\Delta t} s e^{\mathbf{A}_{0}(\Delta t-s)} \mathrm{d} s=\mathbf{A}_{0}^{-1}\left(\boldsymbol{\Phi}_{1}-\Delta t \mathbf{I}\right), \\
& \boldsymbol{\Phi}_{3}=\int_{0}^{\Delta t} s^{2} e^{\mathbf{A}_{0}(\Delta t-s)} \mathrm{d} s=\mathbf{A}_{0}^{-1}\left(2 \boldsymbol{\Phi}_{2}-\Delta t^{2} \mathbf{I}\right),
\end{aligned}
$$

with $\mathbf{A}_{i}^{\langle l, j\rangle}=\mathbf{A}_{l, j}\left(t_{i}\right), \mathbf{B}_{i}^{\langle l, j\rangle}=\mathbf{B}_{l, j}\left(t_{i}\right)$, and $\mathbf{y}_{i}=\mathbf{y}\left(t_{i}\right)$.

where I denotes the identity matrix. 
From (17), it is obtained that

$$
\begin{aligned}
\mathbf{y}_{i+1}-\sum_{j=1}^{N} \sum_{l=1}^{L} \mathbf{P}_{i}^{\langle l, j\rangle} \mathbf{y}_{i+1}= & \left(\boldsymbol{\Phi}_{0}+\sum_{j=1}^{N} \sum_{l=1}^{L} \mathbf{F}_{i}^{\langle l, j\rangle}\right) \mathbf{y}_{i} \\
& -\sum_{j=1}^{N} \sum_{l=1}^{L} \mathbf{F}_{i}^{\langle l, j\rangle} \mathbf{y}_{i-m_{l, j}} \\
& -\sum_{j=1}^{N} \sum_{l=1}^{L} \mathbf{P}_{i}^{\langle l, j\rangle} \mathbf{y}_{i+1-m_{l, j}}
\end{aligned}
$$

By further simplifying, it follows that

$$
\begin{aligned}
(I & \left.-\sum_{j=1}^{N} \sum_{l=1}^{L} \mathbf{P}_{i}^{\langle l, j\rangle}\right) \mathbf{y}_{i+1} \\
= & \left(\boldsymbol{\Phi}_{0}+\sum_{j=1}^{N} \sum_{l=1}^{L} \mathbf{F}_{i}^{\langle l, j\rangle}\right) \mathbf{y}_{i}-\sum_{j=1}^{N} \sum_{l=1}^{L} \mathbf{F}_{i}^{\langle l, j\rangle} \mathbf{y}_{i-m_{l, j}} \\
& -\sum_{j=1}^{N} \sum_{l=1}^{L} \mathbf{P}_{i}^{\langle l, j\rangle} \mathbf{y}_{i+1-m_{l, j}}
\end{aligned}
$$

Let $\mathbf{H}_{i+1}=\left(I-\sum_{j=1}^{N} \sum_{l=1}^{L} \mathbf{P}_{i}^{\langle l, j\rangle}\right)^{-1}, \mathbf{F}_{i}=\sum_{j=1}^{N} \sum_{l=1}^{L} \mathbf{F}_{i}^{\langle l, j\rangle}$, and $\mathbf{P}_{i}=\sum_{j=1}^{N} \sum_{l=1}^{L} \mathbf{P}_{i}^{\langle l, j\rangle}$. Equation (22) can be simplified as follows:

$$
\begin{aligned}
\mathbf{y}_{i+1}= & \mathbf{H}_{i+1}\left(\boldsymbol{\Phi}_{0}+\mathbf{F}_{i}\right) \mathbf{y}_{i}-\mathbf{H}_{i+1} \mathbf{F}_{i} \mathbf{y}_{i-m_{l, j}} \\
& -\mathbf{H}_{i+1} \mathbf{P}_{i} \mathbf{y}_{i+1-m_{l, j}} .
\end{aligned}
$$

As previously defined, $M=\max _{l, j=1,2, \ldots}\left\{m_{l, j}\right\}$. Suppose the maximum value of $m_{l, j}$ can be obtained on the condition of $l=l^{*}, j=j^{*}$; from (23) it follows that

$$
\left(\begin{array}{c}
\mathbf{y}_{i+1} \\
\mathbf{y}_{i} \\
\vdots \\
\mathbf{y}_{i+1-M}
\end{array}\right)=\left(\begin{array}{cccccccc}
\mathbf{H}_{i+1}\left(\mathbf{\Phi}_{0}+\mathbf{F}_{i}\right) & 0 & \cdots & -\mathbf{H}_{i+1} \mathbf{P}_{i}^{\langle l, j\rangle} & -\mathbf{H}_{i+1} \mathbf{F}_{i}^{\langle l, j\rangle} & \cdots & -\mathbf{H}_{i+1} \mathbf{P}_{i}^{\left\langle l^{*}, j^{*}\right\rangle} & -\mathbf{H}_{i+1} \mathbf{F}_{i}^{\left\langle l^{*}, j^{*}\right\rangle} \\
\mathbf{I} & 0 & \cdots & 0 & 0 & \cdots & 0 & 0 \\
0 & \mathbf{I} & \cdots & 0 & 0 & \cdots & 0 & 0 \\
\vdots & \vdots & \cdots & \vdots & \vdots & \cdots & \vdots & \vdots \\
0 & 0 & \cdots & 0 & 0 & \cdots & \mathbf{I} & 0
\end{array}\right) \times\left(\begin{array}{c}
\mathbf{y}_{i} \\
\mathbf{y}_{i-1} \\
\vdots \\
\mathbf{y}_{i-M}
\end{array}\right) .
$$

Let $\mathbf{Z}_{i}=\operatorname{col}\left(\mathbf{y}_{i}, \mathbf{y}_{i-1} \cdots \mathbf{y}_{i-M}\right), \mathbf{Z}_{i+1}=\operatorname{col}\left(\mathbf{y}_{i+1}, \mathbf{y}_{i} \cdots \mathbf{y}_{i+1-M}\right)$, and

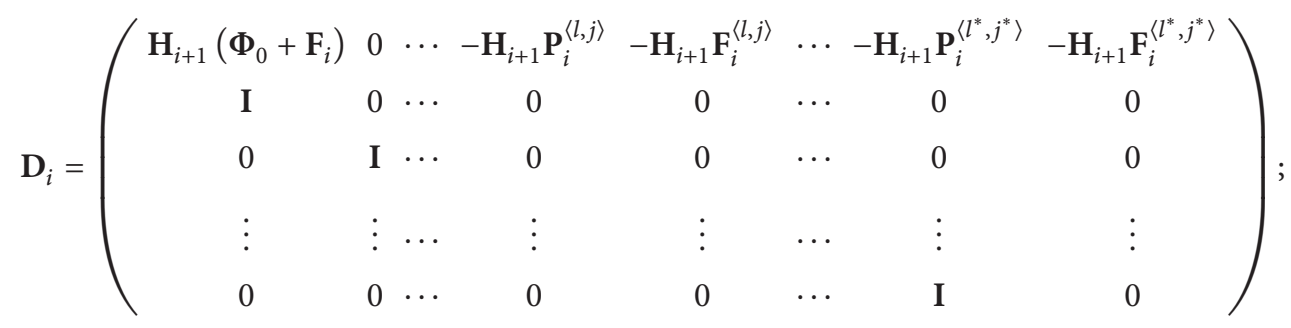

then, $\mathbf{Z}_{i+1}=\mathbf{D}_{i} \mathbf{Z}_{i}$.

The approximate Floquet transition matrix is obtained as $\Phi=\mathbf{D}_{M-1} \mathbf{D}_{M-2} \cdots \mathbf{D}_{0}$.

According to the Floquet theory, the stability of the system is determined using the following criterion. If the moduli of all the eigenvalues of the transition matrix $\Phi$ are less than unity, the system is stable. Otherwise, it is unstable.

\section{Stability Prediction}

To illustrate the correctness of the proposed method, numerical results will be demonstrated under the conditions described in Table 1 . Taking the accuracy into consideration, we choose $k=40 \times N, M=44$, and $L=20$. At the same time, the parameter plane of the spindle speed-depth of cut is divided into a $200 \times 100$ size grid. Stability prediction charts 


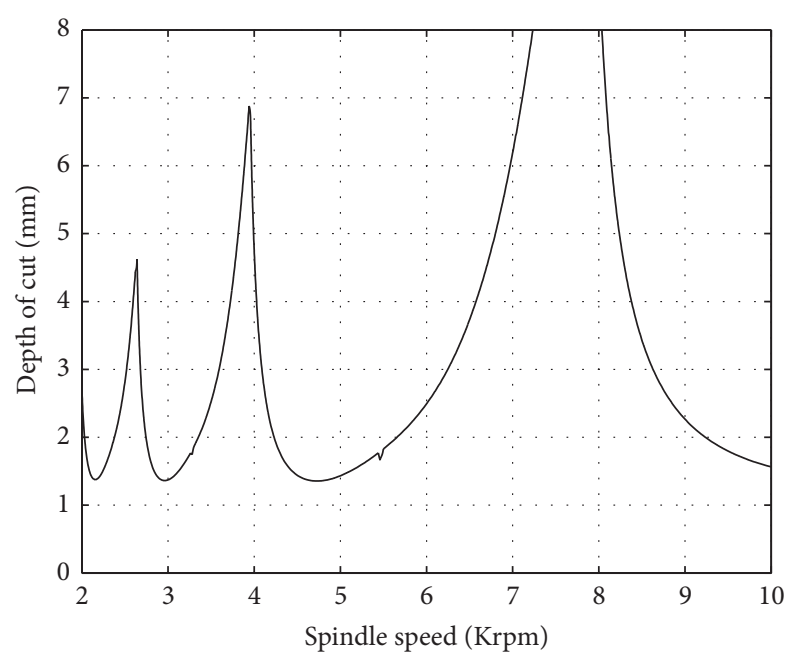

(a)

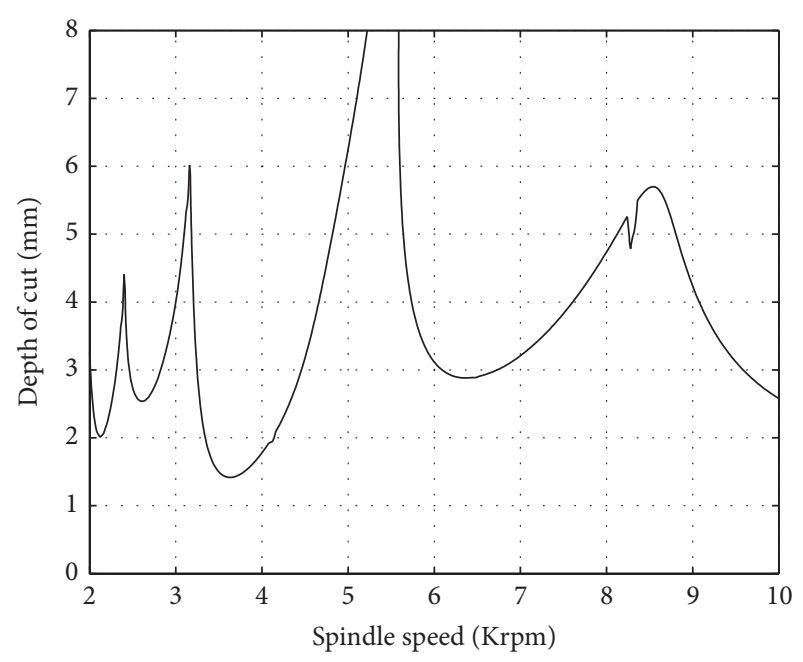

(b)

FIGURE 4: Stability predictions for uniform pitch and variable pitch.

TABLE 1: System parameters.

\begin{tabular}{lc}
\hline Parameter items & Parameter values \\
\hline Number of teeth & $N=4$ \\
Tool diameter [mm] & $d_{c}=19.05$ \\
Radial immersion ratio & $a d_{c}=0.5$ \\
Helix angle [degree] & $\beta=\left[\begin{array}{llll}30 & 30 & 30 & 30\end{array}\right]$ \\
Uniform pitch [degree] & $p=\left[\begin{array}{llll}90 & 90 & 90 & 90\end{array}\right]$ \\
Variable pitch [degree] & $p=\left[\begin{array}{llll}70 & 110 & 70 & 110\end{array}\right]$ \\
Modal masses [kg] & $m_{x}=1.4986, m_{y}=1.199$ \\
Damping ratio & $\xi_{x}=0.055801, \xi_{y}=0.025004$ \\
Natural frequencies [Hz] & $\omega_{n x}=563.55, \omega_{n y}=516.21$ \\
Normal cutting coefficients [Mpa] & $K_{n}=256$ \\
Tangential cutting coefficients [Mpa] & $K_{n}=697$ \\
Milling mode & Down-milling \\
\hline
\end{tabular}

for uniform pitch and variable pitch are shown in Figure 4. It is easy to see from Figure 4 that the stability charts are similar to Figure 6 in [21], which means that the correctness of the proposed method can be guaranteed.

The present section will focus on the study of the stability of variable pitch and variable helix milling. To achieve consistency, we only change the pitch and helix angle; the other parameters are selected the same as the above mentioned. Four groups of the helix angles (i.e., 30-35-30$35^{\circ}, 30-40-30-40^{\circ}, 35-40-35-40^{\circ}$, and $\left.35-45-35-45^{\circ}\right)$ and two groups of the pitch angles (i.e., $70-110-70-110^{\circ}$ and $80-100-80-$ $110^{\circ}$ ) are selected to investigate; computer programs of the proposed method are all written in MATLAB R2010b and implemented on a personal computer [Intel(R) Core(TM) i7-2600, 3.4 GHz, 4GB]. Through the introduction of the algorithm, we aim to explain the fact that the larger stable cutting area can be obtained by variable pitch and variable helix milling. As for the comparison of the different radial immersion ratio, it will not be discussed. Although the radial immersion ratio has a great influence on stability, the omission of the discussion does not prevent the readers from understanding the paper.

The proposed methods of stability charts are presented for variable pitch and variable helix milling (Figures 5 and 6). By comparing Figure 5 with Figure 4(a), it is revealed that the range of stable cutting area for variable pitch and variable helix milling is larger than that for the corresponding uniform pitch and uniform helix milling. In addition, Figures 5 and 4 (b) shows that when spindle speed is $2 \mathrm{Krpm}-4 \mathrm{Krpm}$ for variable pitch tool, there is no significant difference between uniform helix milling and variable helix milling in the range of stable cutting area. However, when spindle speed is $4 \mathrm{Krpm}-10 \mathrm{Krpm}$, stability charts are completely different (i.e., the trends of boundary peaks are exactly opposite). As a result, we can infer that the variable pitch and variable helix milling are more conducive to the chatter suppression in the high-speed region. From the simulation conditions, we know that the pitch angles are the same $\left(70-110-70-110^{\circ}\right)$; the only difference is the helix angle. The variable helix angle leads to delay change in time under the different depth of cut, which disrupts the balance mechanism of the system. This is the real reason of the differences between Figures 5 and 4(b) in the high-speed region. At the same time, it is easy to find that each subgraph of Figure 5 is also different. For each subgraph in Figure 5, the pitch angle is the same, but the helix angle is different (Figure 5(a) is 30-35-30-35 , Figure 5(b) is 30-40$30-40^{\circ}$, Figure 5(c) is 35-40-35-40 ${ }^{\circ}$, and Figure 5(d) is $40-45-$ $\left.40-45^{\circ}\right)$. When the spindle speed is $2 \mathrm{Krpm}-4 \mathrm{Krpm}$, stability lobes in Figures 5(a) and 5(c) are basically the same. However, when the spindle speed is $3.2 \mathrm{Krpm}$, the peaks of the stability boundaries show a little difference in Figures 5(b) and 5(d). When the spindle speed is $4 \mathrm{Krpm}-6 \mathrm{Krpm}$, the peaks of the stability boundaries in Figures 5(a) and 5(c) are greater than Figures 5(b) and 5(d). However, when the spindle speed is $8 \mathrm{Krpm}-9 \mathrm{Krpm}$, the peaks of the stability boundaries in Figures 5(b) and 5(d) are greater than Figures 5(a) and 5(c). 


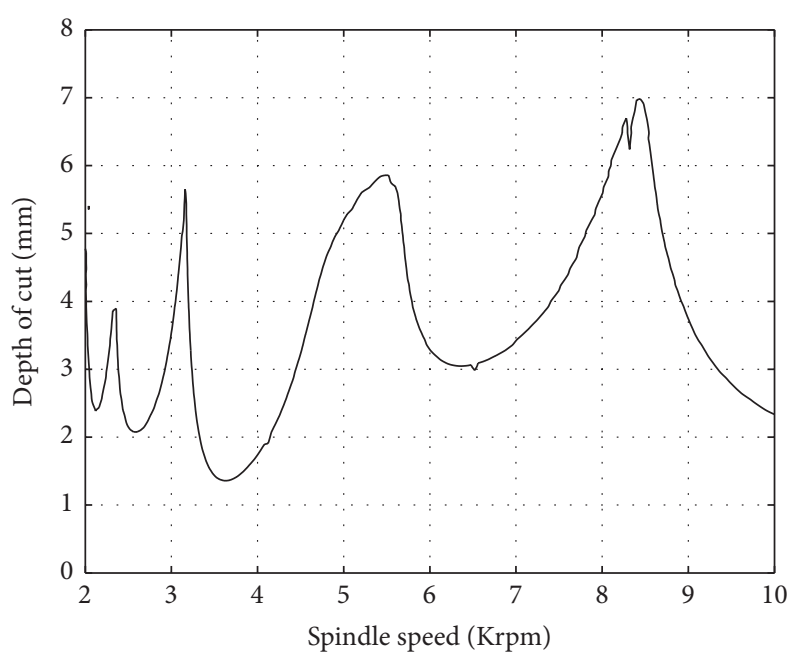

(a) Helix angle $30-35-30-35^{\circ}$

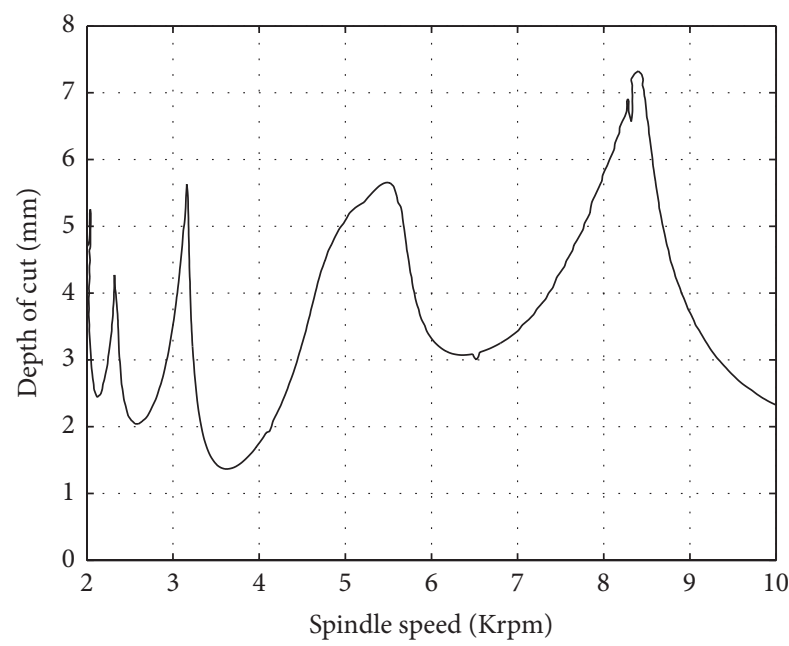

(c) Helix angle $35-40-35-30^{\circ}$

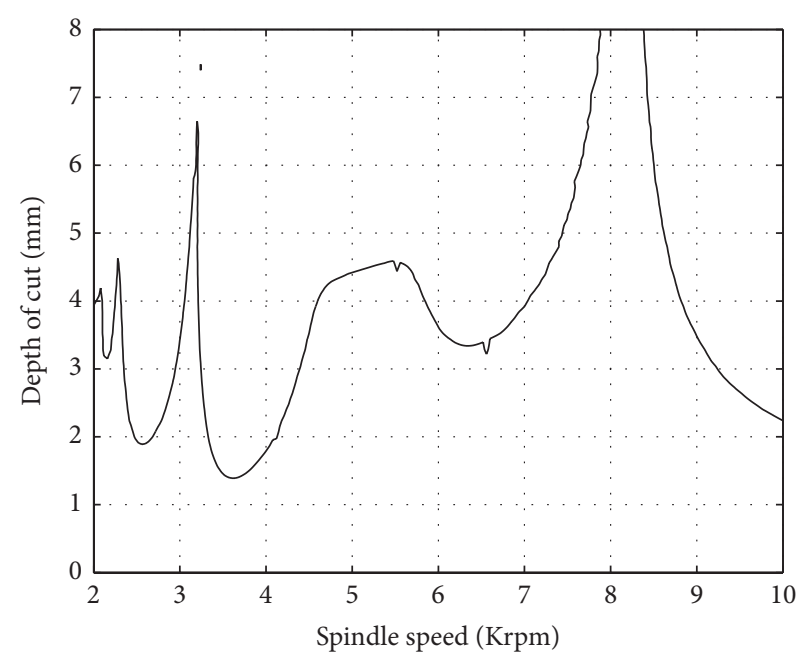

(b) Helix angle $30-40-30-40^{\circ}$

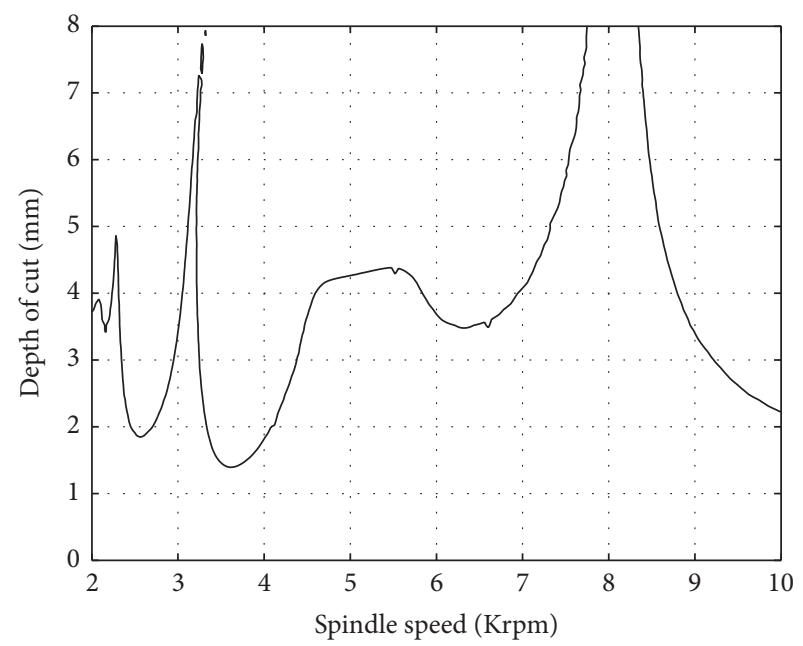

(d) Helix angle $35-45-35-45^{\circ}$

FIGURE 5: Stability charts for 2-DOF down-milling model with pitch angle 70-110-70-110 .

For Figure 6, the pitch angle becomes $80-100-80-100^{\circ}$. For the convenience of comparison, the selection of the helix angle is similar to Figure 5. From Figures 6 and 4(a), it can also be observed that the variable pitch and variable helix milling obtain larger stable cutting area than uniform pitch and uniform helix milling. The main reason is that the cutter has different pitch, which can produce a different phase of the cutting movement to prevent the occurrence of chatter. Thus, it is concluded that chatter can be effectively suppressed by the variable pitch and variable helix milling. In addition, by comparing the corresponding subgraph of Figures 5 and 6, the results show that the lobes in Figure 6 have larger stable cutting area when spindle speed is $2 \mathrm{Krpm}-$ $4.2 \mathrm{Krpm}$. However, the stable lobes in Figure 5 have more advantages when spindle speed is $4.2 \mathrm{Krpm}-10 \mathrm{Krpm}$. The above mentioned conclusion explains that there are large changes for pitch angle (i.e., each tooth pitch angle has 40 degrees' difference in Figure 5) which can contribute to the stability of the milling performance in a high-speed region.
For Figure 6 itself, the stability lobes are exactly the same when spindle speed is $4 \mathrm{Krpm}-10 \mathrm{Krpm}$; there are some small changes only in the low-speed region. When the spindle speed is $2 \mathrm{Krpm}-4 \mathrm{Krpm}$, the cutting stability, as shown in the subgraphs of Figure 6, has its own performance. It is not easy to assert which has more advantages.

Through the above analysis, the variable pitch and variable helix milling are a way to improve the machining accuracy, and this way will also be widely used in the field of machining and plays its due role. In short, the variable pitch and variable helix milling are a feasible and effective method for chatter suppression.

\section{Conclusions}

In this work, an improved semidiscretization algorithm is proposed for variable pitch and variable helix milling. Because the delay between each flute of the variable helix tools varies along the axial depth of the tool, the tool is 


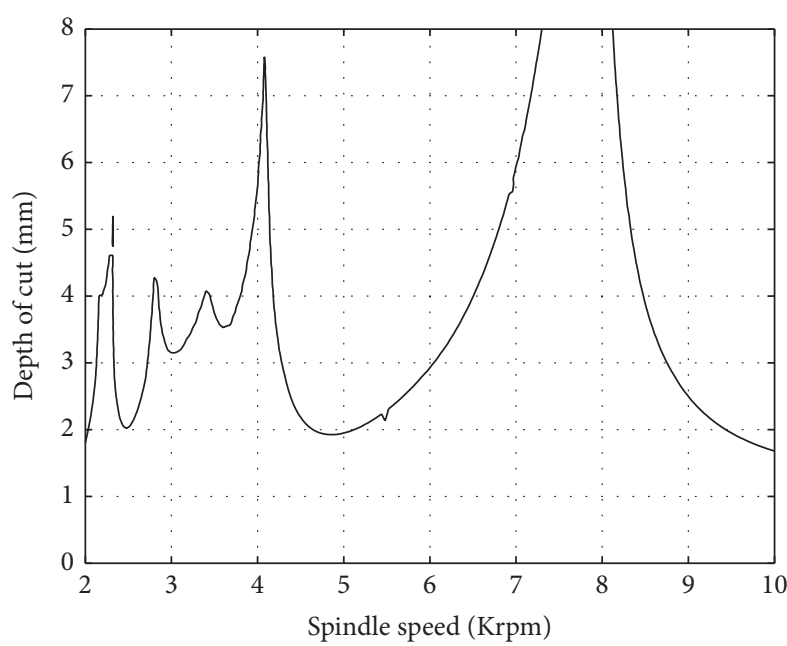

(a) Helix angle $30-35-30-35^{\circ}$

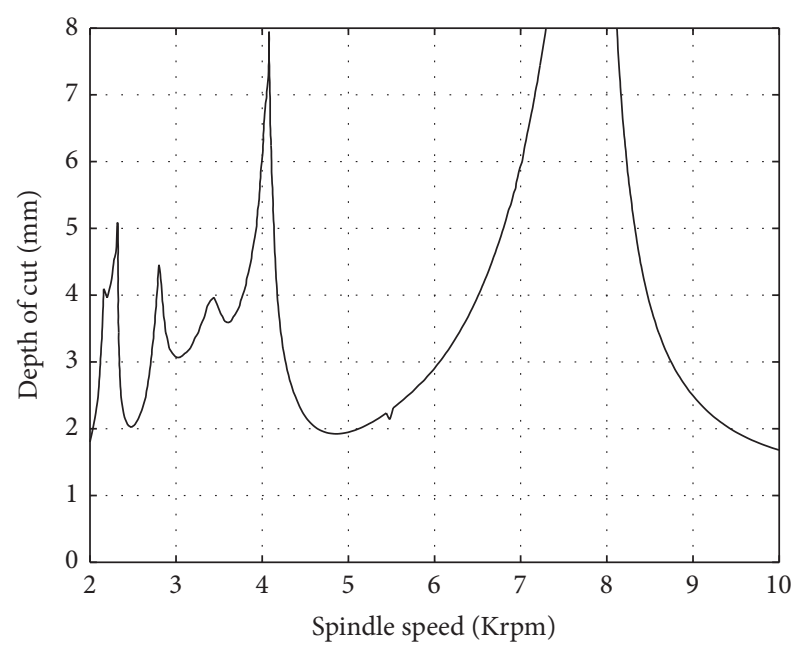

(c) Helix angle $35-40-35-30^{\circ}$

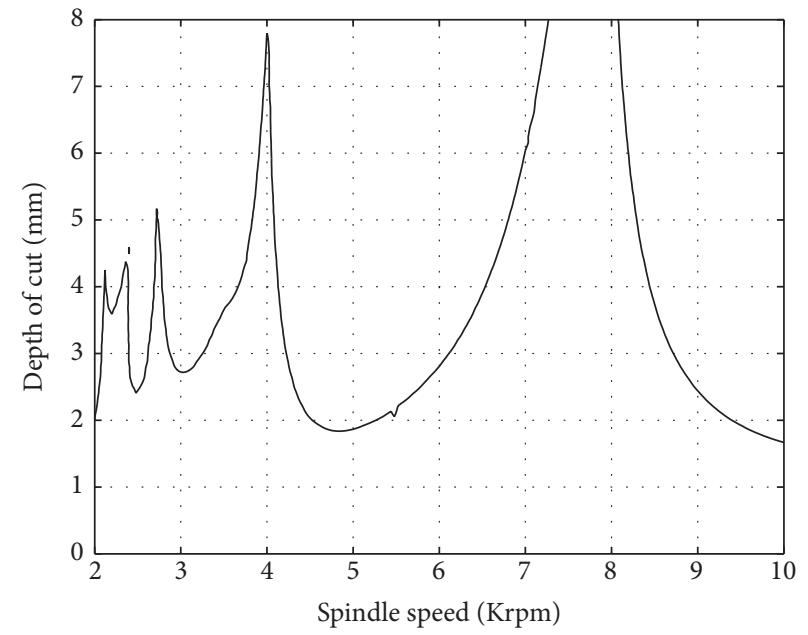

(b) Helix angle $30-40-30-40^{\circ}$

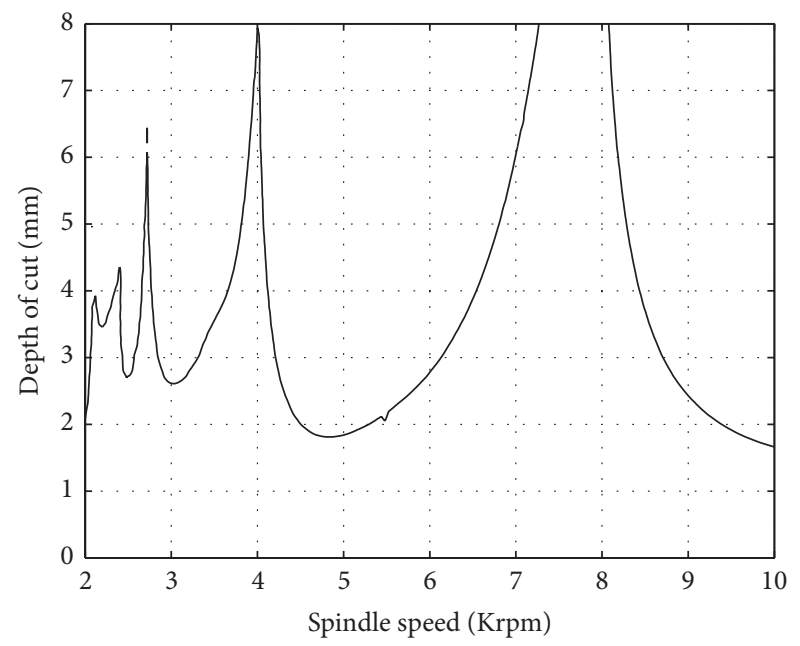

(d) Helix angle $35-45-35-45^{\circ}$

FIGURE 6: Stability charts for 2-DOF down-milling model with pitch angle 80-100-80-100 .

discrete into a number of axial layers to solve this problem. The algorithm is implemented based on the Floquet theory. Two-DOF down-milling models are utilized to demonstrate the algorithm. Meanwhile, the correctness of the algorithm is verified by comparison with the published literature results. In addition, the effects of variable pitch and variable helix milling on the corresponding stability charts are explored; it is revealed that variable pitch and variable helix milling are more effective in suppressing chatter than uniform pitch and uniform helix milling.

\section{Conflict of Interests}

The authors declare that there is no conflict of interests regarding the publication of this paper.

\section{Acknowledgments}

This work is supported by the National Natural Science Foundation of China (51475324), the Natural Science
Foundation of Tianjin (13JCZDJC34000), Tianjin Research Program of Application Foundation and Advanced Technology (14JCZDJC39600), National Key Technology R\&D Program of the Ministry of Science and Technology (2013BAF06B00), and Tianjin City High School Science \& Technology Fund Planning Project (20140408).

\section{References}

[1] Y. Altintaş and E. Budak, "Analytical prediction of stability lobes in milling," CIRP Annals: Manufacturing Technology, vol. 44, no. 1, pp. 357-362, 1995.

[2] Y. Altintas, Manufacturing Automation: Metal Cutting Mechanics, Machine Tool Vibrations, and CNC Design, Cambridge University Press, Cambridge, UK, 2000.

[3] S. D. Merdol and Y. Altintas, "Multi frequency solution of chatter stability for low immersion milling," Transactions of the ASME-Journal of Manufacturing Science and Engineering, vol. 126, no. 3, pp. 459-466, 2004. 
[4] T. Insperger and G. Stepan, "Semi-discretization method for delayed systems," International Journal for Numerical Methods in Engineering, vol. 55, no. 5, pp. 503-518, 2002.

[5] T. Insperger and G. Stepan, "Updated semi-discretization method for periodic delay-differential equations with discrete delay," International Journal for Numerical Methods in Engineering, vol. 61, no. 1, pp. 117-141, 2004.

[6] T. Insperger and G. Stepan, Semi-Discretization for Time-Delay Systems: Stability and Engineering Applications, Springer, New York, NY, USA, 2011.

[7] P. V. Bayly, J. E. Halley, B. P. Mann, and M. A. Davies, "Stability of interrupted cutting by temporal finite element analysis," Transactions of the ASME-Journal of Manufacturing Science and Engineering, vol. 125, no. 2, pp. 220-225, 2003.

[8] Y. Ding, L. Zhu, X. Zhang, and H. Ding, "A full-discretization method for prediction of milling stability," International Journal of Machine Tools and Manufacture, vol. 50, no. 5, pp. 502-509, 2010.

[9] R. P. H. Faassen, N. van de Wouw, J. A. J. Oosterling, and H. Nijmeijer, "Prediction of regenerative chatter by modelling and analysis of high-speed milling," International Journal of Machine Tools and Manufacture, vol. 43, no. 14, pp. 1437-1446, 2003.

[10] S. Yi, P. W. Nelson, and A. G. Ulsoy, "Delay differential equations via the matrix Lambert $\mathrm{W}$ function and bifurcation analysis: application to machine tool chatter," Mathematical Biosciences and Engineering, vol. 4, no. 2, pp. 355-368, 2007.

[11] E. A. Butcher, O. A. Bobrenkov, E. Bueler, and P. Nindujarla, "Analysis of milling stability by the chebyshev collocation method: algorithm and optimal stable immersion levels," Journal of Computational and Nonlinear Dynamics, vol. 4, no. 3, pp. $1-12,2009$.

[12] M. Zatarain, J. Muñoa, G. Peigne, and T. Insperger, "Analysis of the influence of mill helix angle on chatter stability," CIRP Annals-Manufacturing Technology, vol. 55, no. 1, pp. 365-368, 2006.

[13] T. Insperger, J. Munoa, and M. Zatarain, "Unstable islands in the stability chart of milling processes due to the helix angle," in Proceedings of the CIRP 2nd International Conference on High Performance Cutting (HPC '06), Vancouver, Canada, 2006.

[14] B. R. Patel, B. P. Mann, and K. A. Young, "Uncharted islands of chatter instability in milling," International Journal of Machine Tools and Manufacture, vol. 48, no. 1, pp. 124-134, 2008.

[15] Y. Altintaş, S. Engin, and E. Budak, "Analytical stability prediction and design of variable pitch cutters," Transactions of the ASME-Journal of Manufacturing Science and Engineering, vol. 121, no. 2, pp. 173-178, 1999.

[16] E. Budak, "An analytical design method for milling cutters with nonconstant pitch to increase stability, Part I: theory," Journal of Manufacturing Science and Engineering, vol. 125, no. 1, pp. 2934, 2003.

[17] E. Budak, "An analytical design method for milling cutters with nonconstant pitch to increase stability, part 2: application," Journal of Manufacturing Science and Engineering, vol. 125, no. 1, pp. 35-38, 2003.

[18] N. Olgac and R. Sipahi, "Dynamics and stability of variablepitch milling," Journal of Vibration and Control, vol. 13, no. 7, pp. 1031-1043, 2007.

[19] S. Turner, D. Merdol, Y. Altintas, and K. Ridgway, "Modelling of the stability of variable helix end mills," International Journal of Machine Tools and Manufacture, vol. 47, no. 9, pp. 1410-1416, 2007.
[20] A. R. Yusoff and N. D. Sims, "Optimisation of variable helix tool geometry for regenerative chatter mitigation," International Journal of Machine Tools and Manufacture, vol. 51, no. 2, pp. 133141, 2011.

[21] N. D. Sims, B. Mann, and S. Huyanan, "Analytical prediction of chatter stability for variable pitch and variable helix milling tools," Journal of Sound and Vibration, vol. 317, no. 3-5, pp. 664686, 2008. 

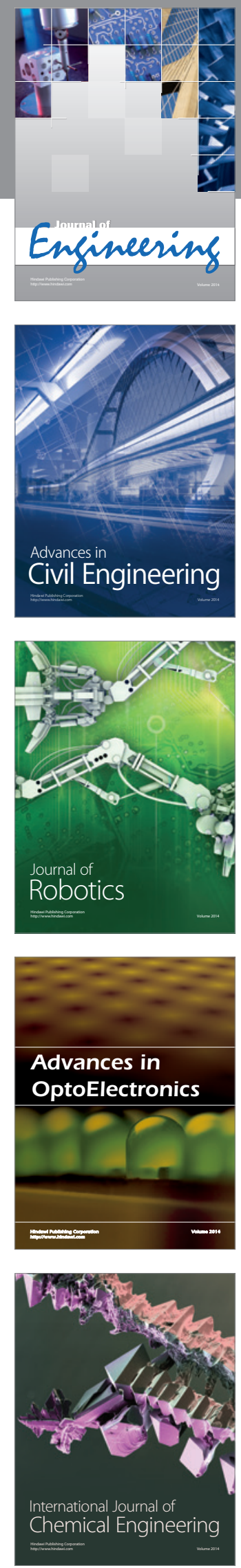

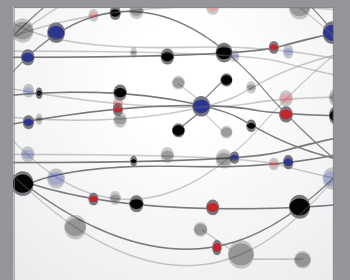

The Scientific World Journal
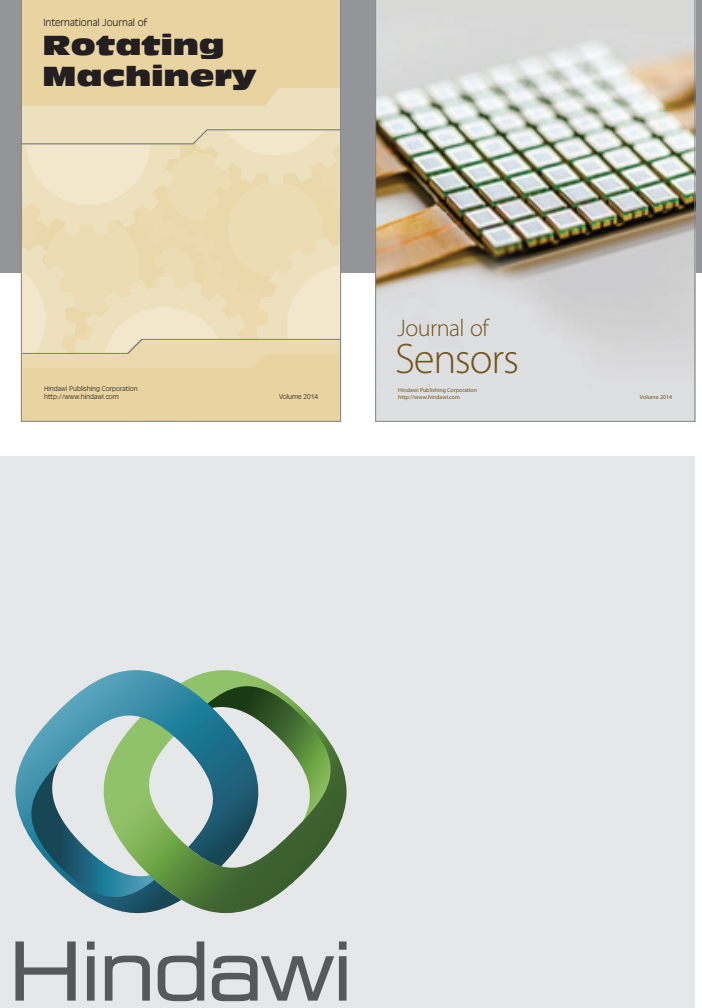

Submit your manuscripts at http://www.hindawi.com
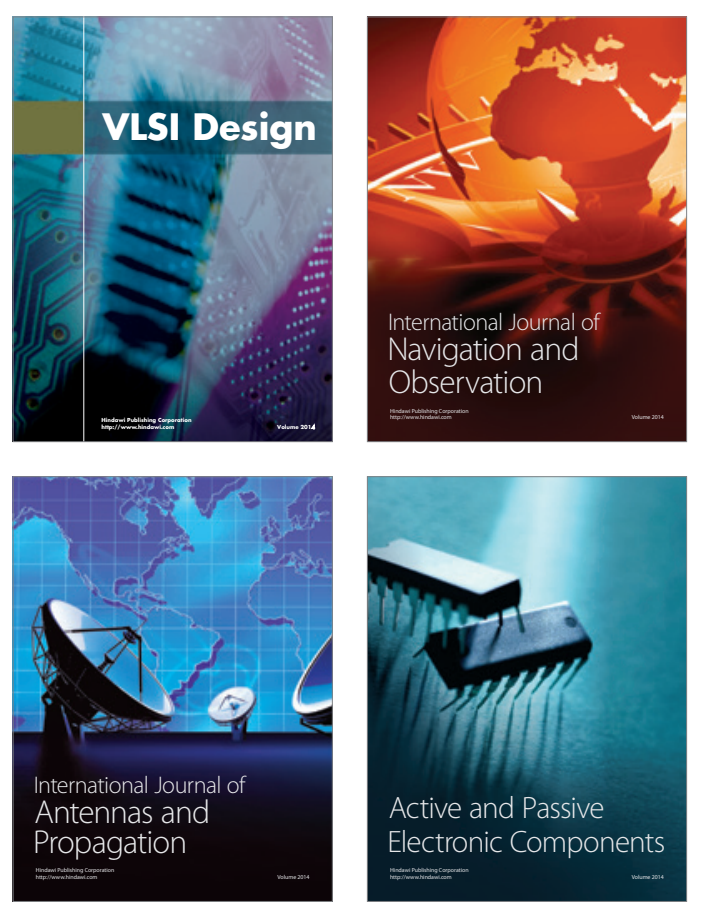
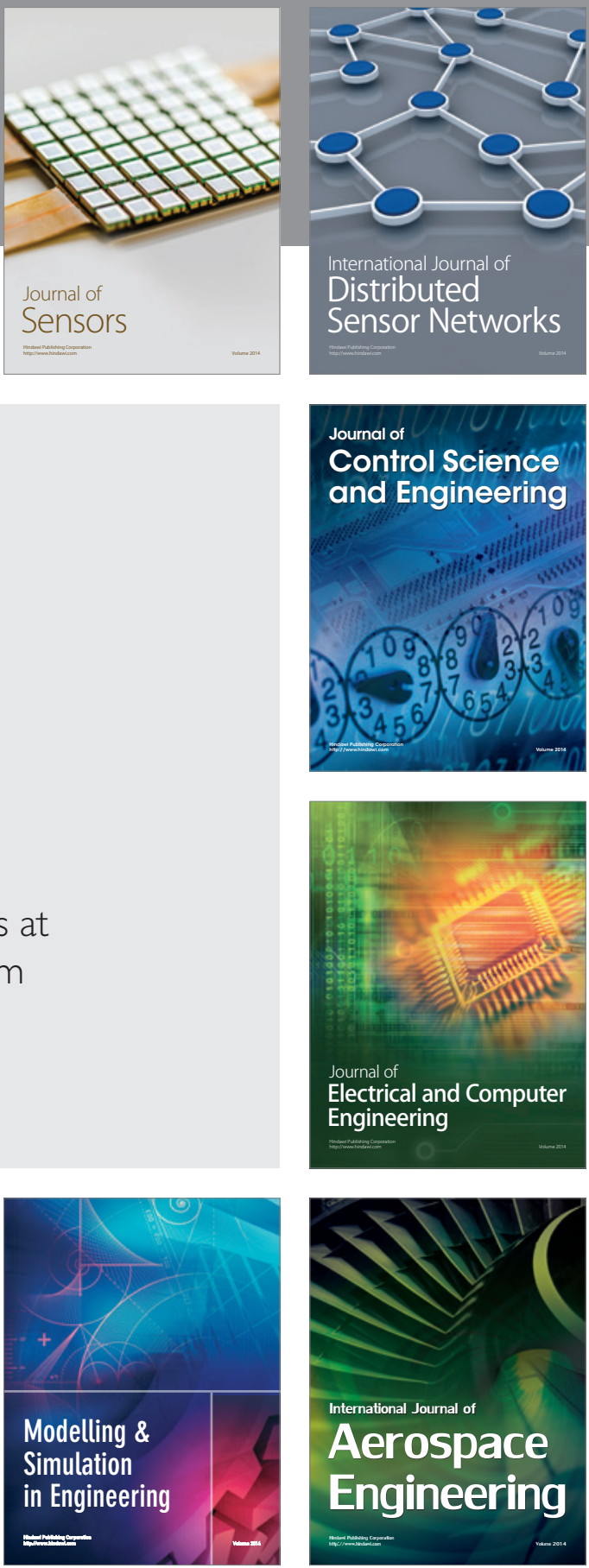

Journal of

Control Science

and Engineering
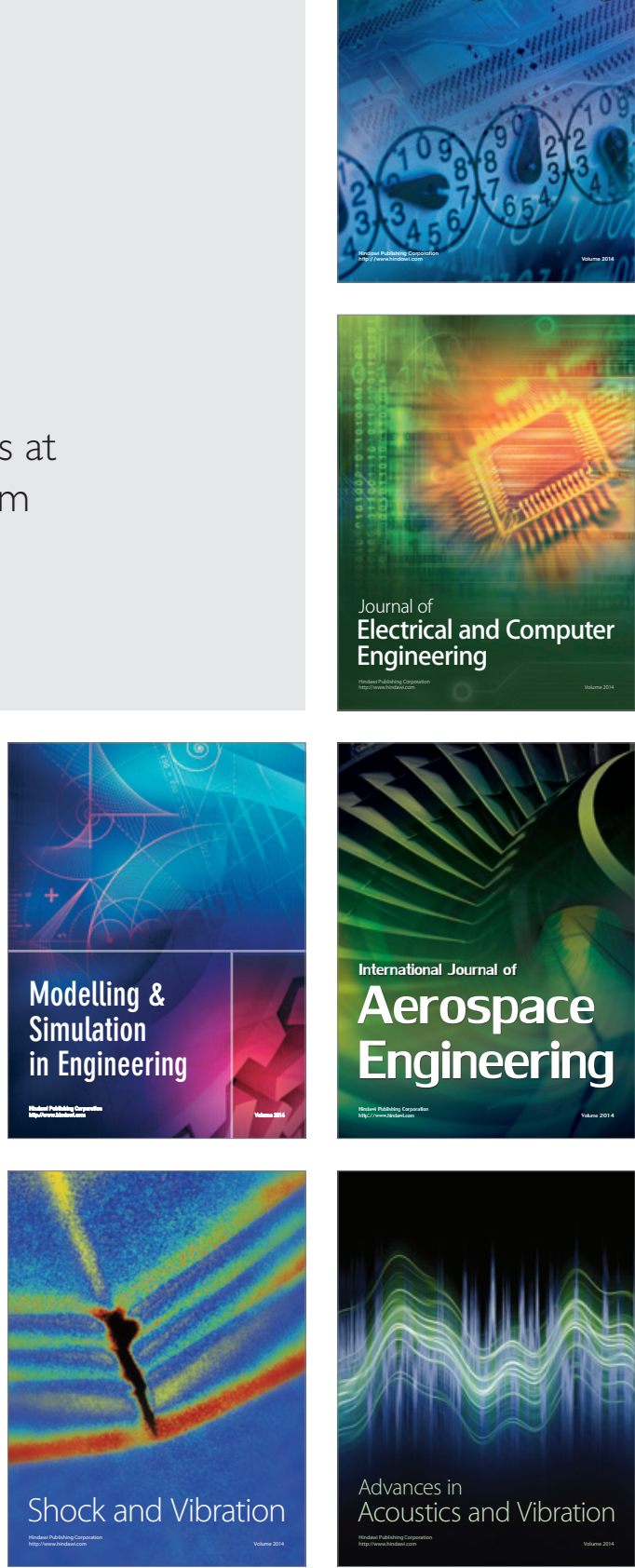